\title{
Apprentissage de la conduite et sécurité routière
}

Un dilemme de référence pour la conception d'un référentiel de diplôme d'enseignant

Teaching driving and road safety: defining a frame of reference for an instructor's diploma based on professional dilemmas

\section{Bernard Prot}

\section{CpenEdition}

\section{Journals}

Édition électronique

URL : http://journals.openedition.org/activites/2599

DOI : 10.4000/activites.2599

ISSN : $1765-2723$

Éditeur

ARPACT - Association Recherches et Pratiques sur les ACTivités

\section{Référence électronique}

Bernard Prot, « Apprentissage de la conduite et sécurité routière », Activités [En ligne], 8-2 | octobre 2011, mis en ligne le 15 octobre 2011, consulté le 30 avril 2019. URL : http://journals.openedition.org/ activites/2599 ; DOI : 10.4000/activites.2599

\section{(c) (i) (2)}

Activités est mis à disposition selon les termes de la licence Creative Commons Attribution - Pas d'Utilisation Commerciale - Pas de Modification 4.0 International. 


\title{
Apprentissage de la conduite et sécurité routière : Un dilemme de référence pour la conception d'un référentiel de diplôme d'enseignant
}

\author{
Bernard Prot \\ Centre de recherche sur le travail et le développement- CNAM \\ bernard.prot@cnam.fr
}

\begin{abstract}
SUMMARY
Teaching driving and road safety: defining a frame of reference for an instructor's diploma based on professional dilemmas. This text provides an approach to the design of professional references based on activity analysis. Based on a study with driving instructors, it tests the hypothesis that such a reference may be based on the formalization of professional dilemmas that come from within a professional genre. First, we present some elements of the institutional context on which two studies were conducted in 2009, and specify the function that the researchers wanted to keep in the process of drawing up the frame of reference, in liaison with the relevant professionals. The analysis of two work sequences provides support for the hypothesis.
\end{abstract}

\section{KEY-WORDS}

Activity, standard frames of reference, dilemmas

L'élaboration d'un référentiel dit «d'activités professionnelles » pour le diplôme des enseignants de la conduite, les moniteurs d'auto-école, est au centre de ce texte. En 2006, les responsables de la Délégation à la Sécurité et à la Conduite Routières (DSCR) ${ }^{1}$, chargés de rénover la filière de formation et mettre à jour les diplômes, sollicitent l'appui de l'équipe de clinique de l'activité à partir des études réalisées sur le fonctionnement de la validation des acquis de l'expérience (Clot \& Prot 2003; Prot, 2011).

Après avoir situé quelques éléments du contexte institutionnel de deux études réalisées successivement de 2006 à 2009, ce texte définit quelques éléments importants de la fonction que les chercheurs ont voulu tenir au regard du processus d'élaboration du référentiel, et de leur coopération avec des professionnels associés. Elle présente alors une approche de la conception de référentiels fondée sur l'exercice du métier en faisant l'hypothèse que cette élaboration pourrait être fondée sur la formalisation de dilemmes spécifiques au genre professionnel. Cette hypothèse, déjà discutée dans deux autres contextes (Clot, Tomás, Kloetzer, \& Prot, 2008 ; Prot, Ouvrier-Bonnaz, Mezza, Reille-baudrin, § Vérillon, 2009) est soutenue ici à partir de l'analyse de deux séquences d'activité d'enseignement de la conduite.

\section{La commande institutionnelle : VAE et « référentiel d'activités professionnelles »}

Le ministère chargé des transports est engagé au premier plan dans les politiques publiques nationales visant à réduire le nombre de morts et d'accidentés sur les routes. Le problème est particulièrement important en France, où l'on comptait 8000 décès à la suite d'accidents routiers en 2002 et environ 4000 au moment de la rédaction de ce texte. Les 15-24 ans sont surreprésentés dans la morbidité et la mortalité routière, puisqu'ils représentent, en 2006, 21 \% des tués sur la route, alors qu'ils constituent

1. Une direction du secrétariat d'État aux transports, ministère de l'Écologie du développement et de l'aménagement durables. 
$13 \%$ de la population ${ }^{2}$. Les acteurs institutionnels du domaine initient et soutiennent de vastes programmes d'études tels que le Programme national de recherche et d'innovation dans les transports terrestres (P.R.E.D.I.T.) ${ }^{3}$. Mais la question est également traitée par de nombreux pays européens, qui ont adopté un programme européen qui reprend à son compte les propositions de chercheurs finlandais (Hattaka, Keskinen, Gregersen, \& Glad, 1999) pour une « gestion théoriquement fondée de la gestion du risque routier des jeunes conducteurs ».

Mais la demande de la DSCR à notre égard vise surtout à tenter de répondre à un tout autre type de problème. Ce ministère administre de longue date des diplômes en matière de sécurité routière et de transport maritime. Il doit les mettre à jour au regard de la loi de 2002, dite « de modernisation sociale », qui a instauré la validation des acquis de l'expérience (VAE) ainsi qu'une instance de régulation des offres de diplômes, titres et certificats en France, la Commission Nationale des Certifications Professionnelles (CNCP), chargée de constituer et de tenir à jour un Répertoire National des Certifications Professionnelles (RNCP) ${ }^{4}$. Cette loi prévoit qu'un diplôme délivré par l'État sera inscrit de droit au répertoire après « avis d'instances consultatives auxquelles les organisations représentatives d'employeurs et de salariés sont parties ». La concertation mentionnée dans la loi porte sur plusieurs questions, telles que les niveaux des diplômes et les correspondances entre les diplômes d'une même filière. Elle doit notamment conduire à établir « une description des activités d'un métier, d'une fonction ou d'un emploi existant, élaboré avec la participation des professionnels concernés » qui sera intégrée au diplôme. Or, le ministère chargé de la conduite routière ne dispose pas d'une telle commission professionnelle consultative.

À la suite de l'adoption de la loi, les services du ministère ont engagé une réflexion pour répondre à ce nouveau cadre national. La DSCR a sollicité le LEST/CNRS ${ }^{5}$ pour explorer la situation de l'emploi dans ce secteur et envisager quelques évolutions possibles (Bouteiller, Dondeyne, Labruyère, \& Mossé, 2005). La DSCR demande un accompagnement du Centre de Recherche sur l'Emploi et les Qualifications (CEREQ) pour étudier les hypothèses d'évolution de la filière de formation et les modalités de certification (Bouteiller \& Labruyère, 2009). Enfin, la DSCR organise une rapide concertation avec les partenaires sociaux pour élaborer un référentiel provisoire, qui est accepté par la CNCP pour deux années, pour permettre le fonctionnement de la certification en attendant qu'une CPC soit saisie et qu'elle ait redéfini un référentiel selon les cadres actuels.

C'est dans ce contexte spécifique que la DSCR conclut une première convention avec notre laboratoire (Clot, Littim, Zittoun, \& Prot, 2007), puis une seconde (Prot, \& Tomàs, 2009) pour soutenir son travail d'élaboration d'un "référentiel des activités professionnelles » des enseignants de la conduite. Le diplôme concerné est alors le Brevet pour l'Exercice de la Profession d'Enseignant de la Conduite Automobile et de la Sécurité Routières (B.E.P.E.C.A.S.E.R.).

\section{Quel référentiel?}

Le terme de référentiel est désormais utilisé dans de très nombreux domaines, aussi convient-il de déterminer de quoi il sera question désormais. Le RNCP contient des diplômes, des titres tels que ceux délivrés par l'Association Nationale pour la Formation des Adultes (AFPA) ou par le Conservatoire National des Arts et Métiers (CNAM), et aussi des Certificats de Qualification Professionnelle (CQP) délivrés par des branches professionnelles. Si le terme de «certifications » les recouvre tous, seul le diplôme est une certification "élaborée ou validée par les autorités publiques et délivrée par l'État ou en son nom » (Caillaud, 2008, p. 78). Une telle légitimité a des conséquences importantes pour le titulaire d'un diplôme, qui peut se prévaloir d' « un certain nombre de droits, garantis par les autorités

2. Chiffres du comité interministériel de la sécurité routière.

3. A l'initiative des Ministères de la recherche, des Transports, de l'Environnement, et de l'industrie, de l'Agence pour le développement de la maîtrise des énergies et l'Agence nationale de valorisation de la recherche.

4. Concernant le fonctionnement du R.N.C.P. on peut se reporter au site http://www.cncp

5. Laboratoire d'économie et de sociologie du travail de l'université de la méditerranée 
publiques » (ibid.).

La loi de 2002 stipule aussi qu'une certification, quelle qu'elle soit, est inscrite au RNCP après «avis d'instances consultatives auxquelles les organisations représentatives d'employeurs et de salariés sont parties ». Dans le cas des diplômes, ce principe est adossé à l'existence de Commissions Professionnelles Consultatives (CPC), au sein du ministère, chargées de donner un avis sur l'opportunité de la création sur la rénovation et la suppression d'un diplôme. Dans le cas des branches professionnelles, c'est la Commission Nationale pour l'Emploi (CNPE) qui constitue l'instance ad hoc. Au moment de l'étude, le ministère chargé des transports ne s'est pas doté d'une telle commission, il envisage plutôt de confier le travail de certification au ministère de l'Éducation nationale, où des CPC fonctionnent de longue date distribuées par branches ou secteurs d'activité (Maillard, 2008), ou bien au ministère chargé du travail qui a institué une CPC à la suite de la loi de 2002 pour gérer, notamment, les titres relevant de l'AFPA. Mais quelle que soit la décision prise, elle devra se situer dans le cadre de la négociation, d'ailleurs rappelée par l'alinéa III de l'article 4 de la loi de 2002 : « Pour un diplôme ou un titre, le dossier de demande d'enregistrement doit comporter : a) une description des activités d'un métier, d'une fonction, d'un emploi, existant et identifié, élaboré avec la participation des professionnels (...)» (J.O. du 28 avril 2002).

On retiendra l'idée que la légitimité du référentiel dont il est question ici est fondée sur l'autorité certificative ainsi que sur l'élaboration du référentiel des activités professionnelles dans un cadre paritaire. Dans cet esprit la fonction des études que nous avons réalisées vise à favoriser « la participation des professionnels concernés » à la négociation, sans se substituer à eux.

Notre approche reprend, prolonge et modifie les travaux classiques en analyse du travail (Béguin, 2007; Clot \& Leplat, 2005; Faïta \& Maggi, 2007; Leplat, 1997, 2000). Ses méthodes d'analyse reposent sur la constitution d'un collectif de professionnels associés aux chercheurs (Clot, Prot, \& Werthe, 2001). De ce point de vue, un référentiel peut se présenter comme des listes de tâches prescrites, au sens considéré en ergonomie comme « un but à atteindre dans des conditions déterminées » (Leplat, 1997, p. 15). C'est le cas par exemple du Référentiel des Activités Professionnelles (RAP) du BEP «métiers du secrétariat » que nous avons étudié dans un autre cadre (Prot, \& Reille-Baudrin, 2007), dont l'arrêté stipule clairement qu'il « analyse les tâches attribuées au titulaire du diplôme et leur contenu dans le cadre de l'évolution du secteur professionnel concerné » (arrêté ministériel du 23 août 1996). Ainsi, l'item 2.2 consacré aux « Activités liées à la gestion de l'information » précise :

« À l'aide de technologies et des méthodes de l'informatique et de la communication et en tenant compte du contexte informationnel et relationnel, des procédures en usages et des consignes reçues :

- collecte, traitement et transmission des informations et données ;

- saisie et mise en forme de l'information quantitative ;

- saisie et mise en forme de l'information textuelle».

Le référentiel du diplôme des enseignants de la conduite qui va nous intéresser (BEPECASER) a été inscrit provisoirement au RNCP avec un référentiel qui relève de cette option. Il est constitué de 30 items successifs, classés en 3 domaines : formation théorique, formation pratique, actions de sensibilisation à la sécurité routière. Les items commencent tous par des verbes d'action, comme ici :

Domaine 1 : Animer des séquences de formation théorique d'éducation à la conduite automobile et à la sécurité routière

- Activité 1: Préparer une séquence de formation théorique collective ou individualisée à l'enseignement de la conduite automobile et de la sécurité routière ;

- Activité 2 : Piloter une séquence de formation théorique collective ou individualisée en tenant compte du programme de formation et de la progression pédagogique du ou des apprenants ;

- Activité 3 : Évaluer la qualité pédagogique d'une formation théorique et en tirer des enseignements. Ces référentiels présentent une limite qui peut être lue depuis la mise en garde qu'Ombredane (1955) 
adressait aux analystes du travail : « trop souvent l'analyseur du travail (...) se plaçant du point de vue normatif, énonce ce que le travailleur doit faire ou est censé faire au lieu de ce qu'il fait réellement» (p. 12). Comment éviter de reproduire, dans la conception des référentiels, cette réduction normative qui peut conduire l'analyse du travail à augmenter encore la liste déjà des normes, buts, méthodes, connaissances et compétences prescrits?

\section{L'exercice du métier et le conflit de normes}

Un retour au concept de tâche peut alors être utile. Le travail confronte à des règles et des obligations souvent nombreuses et qui ne convergent pas spontanément. «Le but privilégié le plus souvent, écrit Leplat (1997), est celui de la production à obtenir (en quantité et qualité, notamment), mais il existe aussi des buts concernant la sécurité (ne pas provoquer d'accident pour soi ni pour les autres), des buts liés à l'activité collective (faciliter le travail des membres de l'équipe) », c'est pourquoi la tâche «peut avoir plusieurs buts, éventuellement conflictuels» (p. 18). Cette question des conflits de buts est très présente dans les travaux d'analyse de l'activité (Caroly, 2002), mais il semble qu'elle soit bien peu présente, sinon absente, dans les référentiels existants.

Elle est importante pour saisir l'intensité et la nature du travail collectif de conception d'une « tâche effective ». Si les conditions et l'histoire du collectif de travail le permettent, les professionnels affectés à une même tâche peuvent développer non seulement une seule et unique manière de réaliser la tâche, mais une gamme plus ou moins variée de réponses qui permettent de prendre en compte les différentes facettes et les aspects contradictoires de la tâche et de la situation présente.

L'idée reprise de Vygotski (1934/1997) que les professionnels développent des « concepts quotidiens » est centrale dans les travaux de clinique de l'activité, mais en la rapportant à « la polyphonie des milieux sociodiscursifs » (Clot, 1999) qu'on trouve dans l'œuvre de Bakhtine et à sa théorie des « genres de discours » (1952/1984). Dans les milieux de travail, l'activité de chacun est confrontée à des divergences de conception qui abondent, et qui sont réglées dans des modes de relations hiérarchiques qui leur donnent une histoire particulière. On dira qu'au travail les concepts quotidiens sont ceux d'un « genre professionnel » (Clot \& Faïta, 2000), constitué de ressources techniques et discursives relativement stabilisées, mais qui peuvent aussi se trouver distribuées dans des variantes plus ou moins nombreuses. De même que les conceptions qui président à l'élaboration de la tâche peuvent être différentes et divergentes, les conceptions élaborées dans un collectif qui reste traversé de dialogues féconds peuvent différer et diverger.

C'est pourquoi notre contribution à l'élaboration de référentiels d'activité ne s'arrêtera pas à tenter de définir ou d'identifier des concepts quotidiens, pas plus qu'elle ne définira des listes de tâches prescrites. L'activité au travail n'est pas assimilable à la mise en œuvre de l'une ou l'autre de ces séries. Pour être efficace, pour gagner en efficience, pour conserver un sens, l'activité doit s'affranchir relativement des prescriptions, comme des manières d'agir et de penser du collectif. L'activité est celle d'un sujet confronté à l'exercice du métier. Le concept de « métier », qui fait l'objet d'une reprise théorique actuellement en psychologie du travail (Clot, 2008), permet de considérer l'activité au travail à travers ces liaisons plus ou moins réussies entre la dimension impersonnelle de la tâche prescrite, celle du genre professionnel qui est transpersonnelle notamment parce qu'elle est distincte de la dimension des relations interpersonnelles qui se tissent directement entre les pairs, avec la hiérarchie et les autres participants à la réalisation du produit ou du service. C'est dans ces relations interpersonnelles qu' on discute ou qu'on renonce à discuter l'organisation de la tâche et les divergences qui surgissent entre pairs ou dans la ligne hiérarchique. Enfin, l'exercice du métier existe à travers la dimension personnelle d'un travailleur, intrapersonnelle plus précisément en ce qu'elle relève de son histoire. L'exercice du métier est «structurellement conflit » (Clot, 2008, p. 258).

C'est pourquoi un concept quotidien qui a d'abord une histoire collective se développe singulièrement, dans un style d'activité auquel il participe à donner une allure. Allons jusqu'à soutenir qu'un 
concept quotidien est essentiellement singulier, c'est là qu'il devient spontané, au sens où il fait corps avec le travailleur. Cette affirmation veut souligner que le concept quotidien est singulier s'il signe un affranchissent subjectif à l'égard de la pensée et de l'action des autres, mais aussi à l'égard de ses ressources antécédentes à partir de ce concept quotidien. C'est ainsi que le concept quotidien fait liaison entre des professionnels qui parviennent à se coordonner parce qu'ils ne pensent et n'agissent pas seulement de la même manière, mais aussi « à titre personnel », avec une possibilité conservée de reprendre l'initiative sur un éventuel dysfonctionnement. En retour, les différences de style d'activité peuvent signaler des divergences de conceptions dans la manière de réaliser l'action, elles peuvent devenir des objets de dialogue professionnels, ce qui participe à les rendre essentiellement collectifs.

On peut alors faire l'hypothèse générale que l'analyse de l'activité réalisée dans un cadre dialogique entre des professionnels permettra de définir des éléments significatifs de l'exercice du métier des enseignants de la conduite pour la conception d'un référentiel.

Ces « éléments » ne sont pas des conceptions de la tâche ou des concepts quotidiens du genre professionnel, ils sont significatifs parce qu'ils provoquent l'occasion ou l'obligation de mobiliser et de relier ces contraintes et ces ressources d'origines différentes. Deux études réalisées dans la même perspective alors que celle-ci se mettait en place ont ouvert une piste de travail pour préciser cette hypothèse, avec des secrétaires (Prot et al., 2008) et avec des cadres syndicalistes (Clot et al., 2008). On a avancé l'idée que ces éléments caractéristiques sont des « dilemmes » et qu'on peut les formaliser pour «matérialiser le rapport, éprouvé par ceux qui s'exposent au travail, entre les contradictions inhérentes aux tâches et les conflits de l'activité » (Prot, 2012; Tomás, \& Prot, 2011). C'est cette hypothèse, dont les termes restent encore provisoires et doivent être mieux définis, que nous pouvons à nouveau mettre à l'épreuve dans l'étude réalisée avec des enseignants de la conduite et de la sécurité.

\section{Réduire pour analyser}

Une première étude d'une durée de 18 mois (août 2006 à février 2008) (Clot, Littim, Zittoun, Prot, \& 2007) s'est déroulée en deux étapes, conclues avec la restitution auprès du comité technique et de la DSCR avec quatre des professionnels ayant participé au travail d'expérimentation :

- La constitution du milieu associé à l'étude, avec les premières observations dans les centres et auprès de 20 enseignants, ainsi que la mise en place d'un comité technique et d'un comité de pilotage. 8 enseignants s'engagent dans les analyses suivantes.

- L'analyse de l'activité d'enseignants. Enregistrement des activités choisies et analyse de ces films avec les professionnels concernés suivant la méthode des autoconfrontations (simples et croisées). Sans revenir sur cette méthode (Clot, Faïta, Fernandez, \& Scheller, 2001; Litim \& Kostulski, 2006; Prot, 2006), on insistera surtout sur le fait qu'elle vise à favoriser l'appropriation du cadre de l'étude par le collectif associé.

La constitution de ce collectif, disposé à s'impliquer à son niveau dans le travail d'élaboration d'un référentiel est en soi un résultat de cette première étude. On veut souligner le travail de réduction opéré par les chercheurs et les professionnels. Les huit professionnels membres du collectif associé à l'étude ont dû choisir ensemble, avec les chercheurs, à travers une série d'échanges de débats, une seule situation de travail qui sera l'objet des autoconfrontations croisées. La contrainte est liée à la méthode - on ne peut pas multiplier les films et les analyses -, mais elle est précisément fondée sur la méthodologie clinique, en ceci qu'on demande aux participants de choisir une situation représentative de nombreux aspects de l'exercice du métier et depuis laquelle on pourra envisager des généralisations. Les professionnels ont opté pour l'enseignement du franchissement d'un rond-point, en considérant, dans leurs premiers arguments, que cette situation réunit trois contraintes très importantes qui sont présentes, mais de manière souvent séparée, dans de nombreuses autres situations :

- L'enseignement du freinage qui pose les questions de l'anticipation, de l'observation de la situation, de l'analyse et du tri des informations, de la prise de décision. Le freinage dégressif, tout 
à la fois geste technique nécessitant une maîtrise de la boîte de vitesse et adaptation de l'allure à la situation, qui nécessite une évaluation des distances avant de s'insérer dans la circulation.

- Le placement du regard de l'enseignant : que regarde-t-il ? Il doit regarder la route et l'élève. Il regarde «partout à la fois », et doit « savoir ce que voit l'élève » pour « enseigner à travers ce que voit l'élève ».

- L'enseignement de la vérification systématique des angles morts. Ce point très important de l'enseignement de la conduite a aussi été repris à partir d'une tendance qui semble se répandre, d'après les participants, chez des inspecteurs qui demanderaient à ne faire vérifier les angles morts « qu'en cas de besoin ». Or, pour les professionnels présents, ce besoin est permanent.

Tout au long de la première étude, et même au cours de la seconde étude, les participants et les interlocuteurs institutionnels ont confirmé la pertinence de ce choix, en considérant qu'il leur permet de penser à bien d'autres situations de conduite ainsi qu'à une très large gamme des objectifs de la formation des élèves, ou encore aux différents niveaux de la matrice européenne GADGET. Finalement, dans le groupe de travail, une expression s'est alors installée : « le rond-point est un carrefour social ».

De notre point de vue on peut considérer cette réduction sur l'activité d'enseignement du franchissement du rond-point comme une condition pour chercher des caractéristiques de l'exercice du métier au sens où nous l'avons défini plus haut. Une telle démarche de réduction demandera par la suite de vérifier la validité plus générale de ces caractéristiques dans d'autres situations et de chercher s'il existe d'autres caractéristiques non présentes dans celle-ci.

À la suite de la restitution des travaux de la première étude, les parties engagent une seconde convention dans cet objectif, intitulée « conception de référentiels et connaissances du métier » (Prot \& Tomás, 2009). Cette convention prévoit l'exploitation des analyses réalisées en autoconfrontation croisée avec les professionnels et aussi avec une commission comprenant des représentants de la DSCR, des institutions professionnelles et des experts. Organisée sur le modèle d'une CPC, sans s'y substituer, cette commission engagerait les débats sur la généralisation des résultats obtenus. La réalisation de cette seconde convention devait coïncider avec les démarches entreprises par le ministère pour qu'une commission professionnelle consultative soit saisie auprès d'un certificateur, le ministère de l'Éducation nationale ou le ministère du Travail étant les plus probables, en vue de la création officielle du nouveau diplôme et de la filière. Le travail réalisé dans le cadre de l'étude pourrait alors être mis à disposition des travaux de la commission consultative.

Mais il a fallu attendre une année après la fin de la première convention pour que la DSCR engage la seconde convention. Ensuite, la DSCR a modifié, reporté ou suspendu les réunions de la commission. Quatre enseignants de la conduite qui avaient participé à la première étude ont malgré tout poursuivi l'élaboration avec les chercheurs, dans l'espoir de rencontrer leurs interlocuteurs. Finalement, sous l'égide de la déléguée interministérielle à la sécurité routière, le dossier de rénovation de la filière est confié dans son ensemble à l'organisme de formation de la branche professionnelle, l'Association Nationale pour la Formation Automobile (ANFA). Cette décision met un terme à l'accompagnement de la rénovation de la filière par le CEREQ, et aussi à notre étude. Ce faisant, le ministère n'a pas mis pas en place les conditions pour valider les résultats et pour envisager leur généralisation. On ne peut pas traiter dans ce texte cette dimension institutionnelle complexe de l'étude. On va délimiter la suite de cet article à l'exposé d'une démonstration pour rendre plus précisément discutable notre hypothèse.

\section{Entre sécurité et enseignement de la conduite : le métier}

La liste des compétences inscrites dans le RNCP est presque exclusivement tournée vers l'action pédagogique. Rapportée aux 30h pendant lesquelles, en moyenne, un élève conduit un véhicule en 
école ${ }^{6}$, cette liste est déjà bien conséquente. Mais dès les premières analyses réalisées dans les écoles de conduites, les deux chercheuses, M. Littim et M. Zittoun, notent les « fortes tensions », et soulignent le « délicat équilibre » que les enseignants doivent trouver entre le fait que l'élève est aussi un client, lui ou ses parents, qui peuvent considérer que les 20 heures exigées par la loi devraient être suffisantes, et ceci dans de petites structures dont les études montrent le fragile équilibre économique (Clot et al., 2008). De même l'enseignant vise à faire en sorte que l'élève sache « se conduire au milieu des autres », en prenant en compte la sécurité routière et la conduite sociale, mais il doit aussi préparer à l'examen. Les chercheuses relèvent aussi que la préparation à l'épreuve théorique, le « code de la route », peut être conçue comme un «pré-requis » à l'apprentissage de la conduite, ou bien comme le pendant nécessaire à l'apprentissage de la conduite. L'organisation du travail d'enseignement peut aussi être réglée différemment sur l'axe d'un dilemme entre le suivi d'un élève par un enseignant ou la spécialisation des moniteurs (par exemple, certains seront plutôt des « finisseurs »). On voit se dessiner ici une approche du travail qui restitue les contradictions inhérentes aux fonctions à remplir et qui laisse place aux dilemmes auxquels les professionnels se trouvent devoir répondre dans l'exercice de leur métier. On va s'en tenir à l'étude de deux prescriptions très présentes ensemble tout au long des analyses de l'enseignement du franchissement du rond-point, et à la définition du dilemme qu'il semble possible d'identifier dans ces situations.

On parlait officiellement des «moniteurs d'auto-école » jusqu'au remplacement du $\mathrm{CAPP}^{7}$ par le BEPECASER, en 1991. Depuis les «enseignants de la conduite automobile et de la sécurité routière » ont remplacé les moniteurs et n'exercent plus en «auto-école », mais dans des « écoles de conduite et de sécurité routières ». Les apprentissages de la conduite et de la sécurité routière apparaissent désormais comme deux fonctions dans le travail de l'enseignant. Lorsqu'on étudie plus précisément le provisoire référentiel qui a été inscrit sur le site du RNCP, on trouve une traduction de cette double mission lors des séquences pédagogiques pratiques. L'enseignant doit :

— «préparer » et «piloter », des séquences de formation pratiques ;

\section{— "Faire preuve d'exemplarité en matière de comportement et assurer la sécurité des personnes} et des biens».

L'enseignant est responsable de la pédagogie il est aussi responsable de la sécurité et doit, à cet égard être « exemplaire ». En réalité, si l'on veut atteindre une sécurité exemplaire avec un véhicule, on a tout intérêt à ne pas laisser un novice prendre le volant. Dans ce cas, il va de soi qu'on ne réalise plus l'objectif de la formation pratique de l'élève. Piloter le véhicule ou piloter la pédagogie sont deux objectifs a priori contraires si on se met à raisonner en termes de sécurité. Les parents qui pratiquent la « conduite accompagnée » avec leurs enfants peuvent se trouver devant cette difficulté et se demander : «est-ce que je reprends le volant ou est-ce que je le laisse encore conduire? » Ils vivent alors la contradiction en eux-mêmes, c'est un conflit de but qui est devenu un conflit d'activité. Mais un parent se lance dans cette difficulté ponctuellement, et si le conflit d'activité devient trop intense, s'il ne trouve pas la manière d'assurer en même temps la sécurité et l'apprentissage, il peut demander à l'enfant de garer le véhicule, pour reprendre le volant dans le passage délicat.

Or, c'est justement là que les enseignants de la conduite font leur métier, à partir de cette contradiction qu'ils doivent parvenir à assumer professionnellement. C'est justement parce que cette contradiction est majeure qu'elle est assurée par des professionnels, qui doivent, pour l'assumer, développer des ressources spécifiques sous la forme d'un dilemme générique. C'est précisément cette hypothèse qu'on veut soutenir depuis deux brèves séquences d'analyse.

La première séquence a été filmée dans des conditions particulières. Lors de la venue des chercheurs pour filmer l'activité de M. Chauveau, l'élève prévue est absente. Une autre élève a été affectée à l'enseignant, mais cette élève n'a pas encore atteint le niveau de la deuxième étape d'apprentis-

6. Un élève doit avoir suivi au minimum 20 heures de leçons de conduite en circulation pour se présenter à l'épreuve pratique du permis de conduire.

7. Certificat d'Aptitude Professionnelle à l'enseignement de la conduite des véhicules terrestres à moteur. 
sage, pendant laquelle, en principe, on commence à s'engager sur des ronds-points. La jeune élève est donc débutante. Mais on décide, d'un commun accord, de réaliser une leçon qui passe par un rond-point. Il n'y a donc pas lieu de se méprendre sur ce qui suit : cette séance ne représente pas le travail « ordinaire » de l'enseignant de la conduite, elle est construite en partie pour les besoins de la recherche. Son intérêt est que les difficultés de la débutante mettent en évidence l'action de l'enseignant confronté au dilemme entre pédagogie et sécurité, qui serait sans doute moins observable si l'élève était plus avancée.

L'élève s'est engagée sur un rond-point à deux voies après avoir roulé près du terre-plein central, sur la voie de gauche, elle déplace progressivement son véhicule vers la voie de droite, parce qu'elle doit sortir sur la prochaine rue. Elle arrive devant l'entrée qui précède sa propre sortie. Elle tourne alors la tête franchement vers la droite, pour s'assurer qu'aucun véhicule n' arrive dans l'angle mort. Mais ses mains suivent un peu son mouvement de tête, le véhicule se rapproche trop du trottoir. L'enseignant pose alors la main sur le volant, pour contrôler la trajectoire, simultanément, il dit « dernier contrôle à ta gauche ». En même temps qu'il donne cette consigne, il montre du doigt l'angle mort gauche, invitant l'élève à changer complètement l'axe de son regard. Il est en effet toujours possible qu'un véhicule, bien qu'il n'en ait pas le droit, vienne directement du centre du rond-point et se rabatte juste devant l'auto-école, pour sortir lui aussi sur la même rue. C'est une mesure de sécurité. L'enseignant donne la consigne d'une voie calme, corrige légèrement la direction et vérifie lui-même les deux angles morts et le dégagement de la route vers l'avant. Le tout dans un même mouvement, sans précipitation.

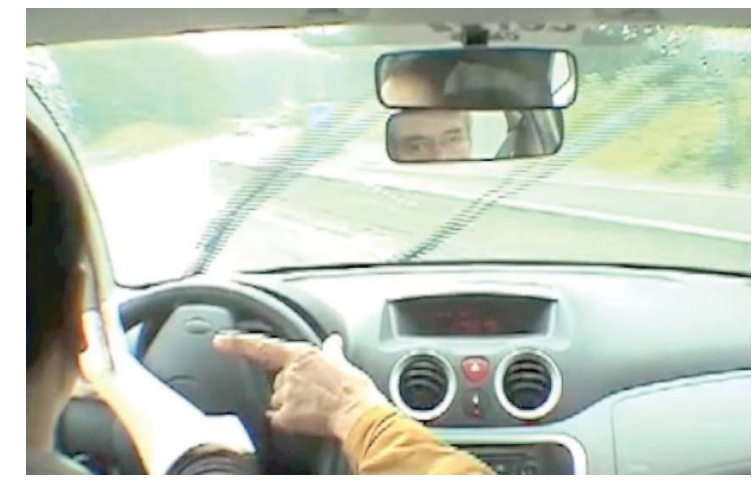

L'enseignant assure à la fois le pilotage pédagogique et le pilotage du véhicule, qu'il partage avec l'élève. Le moniteur est parvenu à unifier les deux termes de la tâche prescrite dans un geste qui intègre les différents objectifs. On retrouve ici l'idée qu'un concept quotidien est complexe en ce sens qu'il y entre des éléments d'ordres différents et qu'il s'est développé dans un seul geste, sans qu'il soit utile d'avoir conscience des ressources multiples mobilisées (Prot 2011a; Vygotski, 1934/1997). Ici l'action porte simultanément sur le véhicule et sur l'élève, le guidage gestuel est coordonné avec un guidage verbal. Ces guidages verbaux et gestuels sont d'ailleurs eux-mêmes intégrés dans une technique pédagogique préméditée par l'enseignant, puisqu'il a annoncé pendant les explications données en stationnement avant le départ : « je vais te guider ». Ce geste complexe unifie, il semble fluide à l'image, il paraît simple à réaliser, sans à coup, ce qui est sans doute rassurant pour l'élève. D'ailleurs dès la sortie du rond-point l'enseignant passe à la priorité suivante, sans attendre : «Allez, accélère! ». Lorsqu'on sort d'un rond-point, il faut éviter d'encombrer la sortie de ceux qui s'y trouvent encore engagés.

La contradiction qui existait initialement dans la coexistence de deux tâches différentes est prise en charge. Le dilemme n'a pas disparu, il faut toujours piloter le véhicule et piloter le travail pédagogique, on ne peut renoncer à l'un ni à l'autre. Mais les ressources professionnelles permettent de relier les deux termes du dilemme et d'agir professionnellement, de réaliser le double «pilotage » prescrit. 
Mais dans cette situation l'élève est débutante. Il n'est pas question de tenir ainsi le volant encore plusieurs leçons. L'objectif est de développer l'autonomie de l'élève. C'est ce qui sera discuté dans les séquences suivantes en considérant que le dilemme ne s'estompe pas lorsque l'élève gagne en maîtrise du véhicule. La vitesse est plus grande, les risques plus élevés, d'autant que l'enseignant engage l'élève dans des conditions de circulations plus complexes, plus exposées. Le dilemme est seulement moins apparent, parce que l'action sur le véhicule est plus inhibée, au profit d'une attention aux conditions de circulation présentes et à la suite du circuit, aux réactions de l'élève. La conduite du véhicule devient, pour le moniteur, une « conduite intérieure » à mesure que l'élève prend le véhicule en main. Alors que la conduite pédagogique, quant à elle, passe moins par les gestes, elle se réalise à travers des modalités langagières, elle se déploie dans la dimension inter-psychologique entre l'enseignant et l'élève.

L'action pédagogique peut aussi se déployer en salle, avant de monter dans le véhicule, comme le montre la séquence suivante, sur laquelle on lira une divergence de méthode entre deux enseignants.

Les enseignants ont été filmés lors d'une séance théorique en salle, précédant le départ en véhicule vers une série de ronds-points. L'enseignant, B. Galland, utilise un tableau blanc devant quelques élèves qui vont partir ensemble dans le véhicule. Il s'agit pour lui de préparer les élèves avant de les engager sur le franchissement d'une série de ronds-points. Il précise à l'aide de schémas les différentes manières de traverser et les imprévus possibles. Il arrive alors au moment de la sortie, lorsque l'élève se trouve devant la sortie qui précède celle qu'il empruntera.

«Alors, le petit danger, il commence ici. [en traversant devant une sortie] Tu vois, tu as ton clignotant à gauche, puisque tu veux sortir là [à la sortie suivante]. Et le danger, tu le comprends, c'est celui qui te couperait la route! Cassandre ! Ça fait déjà deux fois qu'elle te le fait... [La figure de Cassandre a été évoquée l'instant d'avant] D'accord? Elle te coupe la route! Alors là tu as intérêt à faire plus que la moitié. Alors tu as intérêt à jeter un coup d'œil au-dessus de ton épaule gauche pour t'assurer qu'il n'y en a pas un qui va te couper la route! ».

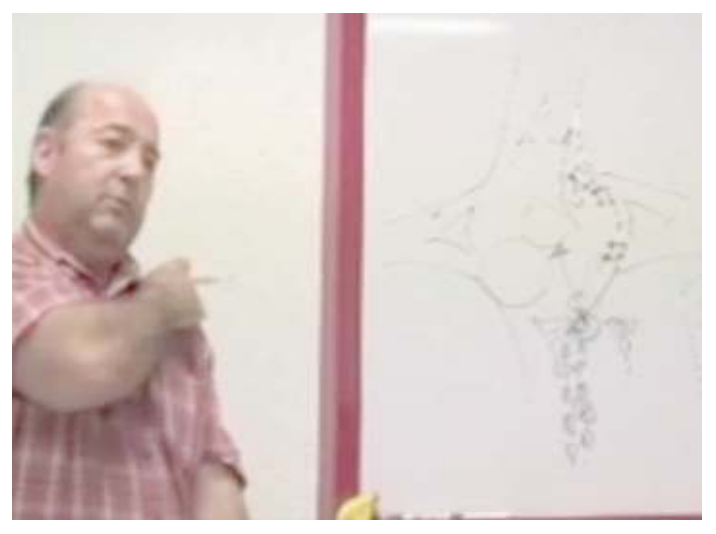

C'est pour répondre au même dilemme que l'enseignant a inventé toute une petite dramaturgie pédagogique devant le tableau, sur fond du mythe de Cassandre, pour sensibiliser les élèves à la difficulté avant qu'ils ne se trouvent dans la situation. Mais devant cet enregistrement, en autoconfrontation croisée, un troisième enseignant, $\mathrm{M}$. Tepper, réagit sans détour :

«M. Tepper : Non, ça ne m'arrive jamais. Ça ne m'arrive jamais, parce que je ne sais pas si tu as vu tout à l'heure, je parle énormément en voiture et je guide énormément. Donc quand je vois cette situation, je vais prévenir les élèves, je vais dire "là tu ne peux pas changer de voie, tu vas rester à droite". Donc je vais anticiper sur l'action que pourrait faire l'élève. C'est-à-dire que là je vais faire du guidage et je vais lui expliquer en étant déjà ici, là "tu vois tu ne peux pas changer de voie".

B. Galland : On est d'accord, on est d'accord... mais là je ne suis plus dans le guidage, là. Là je suis dans le guidage, là [en salle]. Mais tout à l'heure, quand je travaillerai - pas dans l'immédiat, mais 
quand je vais opérationnaliser l'exercice - je ne vais pas être dans le guidage. Et si je propose une solution comme celle-là, je sais, c'est ce que beaucoup vont faire.

M. Tepper : Alors que moi, je reste dans le guidage. Je reste encore dans le guidage.

B. Galland : Mais dans l'opérationnalisation, je ne vais pas rester dans le guidage. C'est à elles [les élèves] de me reproduire les exercices qu'on a effectués, qu'on a travaillé, il va falloir...

$(\ldots)$

M. Tepper : Oui, là tu as pratiqué le système : « là je donne tant d'exercices. À la fin vous en réussissez tant... » On valide le...Moi ce principe-là, je ne l'applique pas, je ne l'applique pas !

B. Galland : Là on était en cours... on voit comment on procède de $\mathrm{A}$ à Z.

M. Tepper : Là moi de "A à Z", je ne pratique pas comme ça.

Chercheuse : Comment vous pratiquez alors?

M. Tepper : Moi je vais l'aider, je vais le guider jusque vraiment je sente qu'il a acquis. Une fois qu'il a acquis, je vais le lâcher. Mais ensuite, je ne vais pas faire ces exercices, "je valide... Tu fais 5 ronds-points, si sur les 5 tu en réussis 3 c'est bien!'. Ça je ne fais pas. Je ne le fais pas. Parce que je n'ai pas envie de le faire. C'est une manière de faire que je... On la fait maintenant avec les livrets de formation, que je ne juge pas très utile. On n'est pas là pour faire un jeu. "Tu as réussi, tu n'as pas réussi...". On voit. Tant que je vois qu'il n'a pas réussi, je vais lui expliquer. Ensuite, quand je vois qu'il sait le mettre en pratique, je vais le laisser faire, je vais le lâcher. Et s'il me fait une erreur, un jour ou l'autre, bon je vais lui ré-expliquer à ce moment-là. Mais je ne vais pas faire des petits jeux "tu réussis". Non. Je vais lui dire à la fin "c'est bien tu as vu, tes ronds-points aujourd'hui ils étaient réussis". Je vais le féliciter à la fin pour les ronds-points qu'il a bien pris. C'est tout. Mais je ne vais pas faire un petit jeu de 7 sur 7 voilà ! Ça je ne le fais pas moi. (BG : non, non, ça ce n'est pas...) Mais je vais le guider. Tant qu'il n'a pas réussi, je vais le guider. »

La controverse est lancée sur la manière de parvenir à tenir ensemble les deux impératifs de sécurité et d'apprentissage. La conception plus ou moins unifiée par laquelle chacun avait réglé son activité se trouve remise en cause, au sens premier du mot, dans le dialogue collectif. La manière de conjuguer sécurité et pédagogie, développée vers l'action, est entrée ici dans une nouvelle zone de développement, celle du dialogue entre pairs.

Deux options pédagogiques s'opposent, lorsqu'il s'agit de trouver la manière d'enseigner la conduite et simultanément de conserver la maîtrise du véhicule en sécurité. Comment répartir les moments de « guidage » et les moments de conduite? Pour B. Galland, dans cette séance, le guidage est principalement réalisé en salle, puis vient la conduite sans guidage, ou du moins c'est schématiquement l'argument avancé. Pour M. Tepper, le guidage verbal est constant pendant la conduite. On n'en déduira pas que cette argumentation est la reproduction de leur activité en situation. Le point central est dans le fait que l'un et l'autre ouvrent une argumentation qui place l'exercice du métier dans l'exercice dialogique entre pairs, en remettant au travail les styles professionnels de chacun. Le dilemme initial est complété d'une série de possibilités différentes.

Ces séquences successives montrent trois manières de prendre en charge cette même contradiction, trois ressources différentes. Ces variations nous font penser que la contradiction initiale est transformée en dilemme générique, il s'agit bien d'un même et unique dilemme entre sécurité et pédagogie, il est spécifique à ce genre professionnel, il ne reste pas à l'état de conflit d'activité parce qu'il est assorti de ressources professionnelles pour y répondre, des ressources que ce dilemme suscite et que le dialogue peut renouveler, s'il est conduit depuis ce dilemme.

\section{Conclusion}

L'étude présentée ici apporte quelques confirmations à l'idée qu'une approche par les dilemmes gé- 
nériques est intéressante à approfondir, pour l'élaboration de référentiels de diplômes professionnels. Un dilemme n'est pas de même nature qu'une tâche, ou un concept quotidien. Il relève de l'exercice du métier, lorsque le professionnel doit relier ensemble des tâches, buts et méthodes prescrits qui peuvent être contradictoires entre eux, et aussi des manières d'agir et de penser issues de l'histoire collective, ou encore de la formation professionnelle, qui ne s'accordent pas plus spontanément.

En procédant à une formalisation d'un dilemme caractéristique de l'exercice d'un métier, on n'identifie pas ce métier à telle ou telle norme d'action, on ne le disperse pas dans des listes de fonctions ou d'unités séparées, on permet aux professionnels de soutenir le dialogue sur diverses possibilités d'action, sans fermer la possibilité d'en envisager de nouvelles.

Le dilemme est générique quand il est spécifique aux professionnels d'un même genre. Il suscite le sentiment d'une difficulté non résolue et qui ne sera pas résolue par un simple choix entre l'un des deux termes, une difficulté qui trame historiquement le genre professionnel parce qu'elle relève des fonctions qu'on doit justement assumer lorsqu'on appartient à ce genre professionnel. Il constitue un stimulateur du dialogue professionnel, à propos duquel il reste à dire, à penser, à inventer, il suscite le développement du métier de chacun en portant les manières d'agir et de penser jusqu'à leurs limites.

C'est pourquoi cette piste de travail, qui doit encore être approfondie sur bien des plans, en commençant par le concept de dilemme, semble aussi pertinente pour organiser le dialogue dans les instances paritaires, ces instances dans lesquelles la discussion sur l'exercice du métier et son avenir constituent en principe la condition et le moyen d'élaborer des référentiels.

\section{RÉFÉRENCES}

Bakhtine, M. (1952/1984). Esthétique de la création verbale. Paris: Gallimard.

Béguin, P. (2007). Dialogisme et conception des systèmes de travail. In Y. Clot \& K. Kostulski « Dialogue, activité, développement », Psychologie del'interaction, n 23-24, 169-199.

Bouteiller, J., Dondeyne, C., Labruyère, C., \& Mossé, P. (2005). Métiers et filières de formation de la sécurité routière. Une analyse en région PACA. Rapport LEST/ORM /CEREQ. [en ligne :www.lest.cnrs.fr/ IMG/.../2003-2006_maj_30-05-2007.doc]

Bouteiller, J., \& Labruyère, C. (2009). La refonte de la filière de formation professionnelle des enseignants de la conduite et de la sécurité routière. Net.doc, 58. [en ligne : www.cereq.fr/pdf/Net-Doc-58.pdf]

Caillaud, P. (2008). Vers une normalisation juridique de la certification professionnelle? In F. Maillard (ED.), Des diplômes aux certifications professionnelles. Nouvelles normes, nouveaux enjeux (pp. 75-96). Rennes: PUR.

Caroly, S. (2002). Différences de gestion collective des situations critiques dans les activités de service selon deux types d'organisation du travail. Pistes, 4(1). http://www.pistes.uqam.ca/v4n1/sommaire.html

Clot, Y. (1999). Avec Vygotski. Paris: La Dispute.

Clot, Y. (2008). Travail et pouvoir d'agir. Paris: PUF.

Clot, Y., \& Faïta, D. (2000). Genres et styles en analyse du travail. Concepts et méthodes. Travailler , 4, 7-42.

Clot, Y., Faïta, D., Fernandez, G., \& Scheller, L. (2001). Les entretiens en autoconfrontation croisée : une méthode en clinique de l'activité. Éducation Permanente, 146, 17-27.

Clot, Y., \& Leplat, J. (2005). La méthode clinique en ergonomie et en psychologie du travail. Le Travail Humain, 68(4), 289-316.

Clot, Y., \& Prot, B. (2003) (Eds.). De l'analyse du travail à la validation des acquis. L'Orientation Scolaire et Professionnelle, 2.

Clot, Y., Prot, B., \& Werthe, C. (2001). Clinique de l'activité et pouvoir d'agir. Éducation permanente, 146. 
Clot, Y., Littim, M., Zittoun, M., \& Prot, B. (2007-2008). Éducation à la conduite et à la sécurité routière. Rapport d'étude, Ministère de l'Équipement, Direction de la conduite et la sécurité routière. Paris.

Clot, Y., Tomás, J.L., Kloetzer, L., \& Prot, B. (2008). Des dilemmes du travail syndical au référentiel. La $V A E$ à la CFE-CGC. Rapport d'étude à la CFE-CGC.

Faïta, D., \& Maggi, B. (2007). Un débat en analyse du travail. Toulouse: Octarès.

Hatakka, M., Keskinen, E., Gregersen, N.P., \& Glad, A. (1999). Théorie et objectifs des mesures d'éducation et de formation. Dans BFU, Vers une gestion théoriquement fondée du risque routier des jeunes conducteurs. Résultat du projet européen GADGET. Groupe de travail N 3 . Berne: Siegrist Editeur.

Leplat, J. (1997). Regard sur l'activité en situation de travail. Contribution à la psychologie ergonomique. Paris: PUF.

Leplat, J. (2000). L'analyse psychologique de l'activité en ergonomie. Aperçu de son évolution, ses modèles et ses méthodes. Toulouse: Octarès.

Maillard, F. (2008). De la démocratisation de l'enseignement et de la formation à la certification généralisée. In F. Maillard (Ed.), Des diplômes aux certifications professionnelles. Nouvelles normes et nouveaux enjeux (pp. 45-73). Rennes: Presses Universitaires.

Ombredance, A. (1955/2001). L'analyse du travail. Facteur d'économie humaine et de productivité. In J. Leplat (Ed.), L'analyse du travail en psychologie ergonomique. Recueil de textes (pp. 9-22). Toulouse: Octarès.

Prot, B. (2006). La fonction du clinicien dans les méthodes de clinique de l'activité. Nouvelle revue de psychosociologie, I, 31-34.

Prot, B. (2011). Connaissances du travail et reconversions professionnelles. In B. Maggi (Ed.), Interpréter l'agir : un défi théorique (pp. 203-219). Paris: PUF.

Prot, B. (2012) (à paraître). «Les dilemmes génériques dans la conception des référentiels d'activité professionnelle : une piste de travail. In F. Maillard (Ed.), Former, certifier, insérer. Effets et paradoxes de l'injonction à la professionnalisation des diplômes.Rennes: PUR.

Prot, B. (Ed.), Ouvrier-Bonnaz, R., Mezza, J., Reille-baudrin, E., \& Vérillon, P. (2009). Développer le métier pour rénover le référentiel. Une étude réalisée avec des employés administratifs et des enseignants de BEP « métiers du secrétariat ». CPC Documents, 8. Paris: MEN.

Prot, B., \& Reille-baudrin, E. (2007). Entre le référentiel et l'activité : le problème de la prospective du métier. Une étude avec des employés de bureau. CPC documents, 1.

Prot, B., \& Tomàs, J.L. (2009). Concevoir un référentiel à partir de l'exercice du métier. Avec des enseignants de la conduite automobile. Rapport à la Délégation à la Sécurité et à la Circulation Routières, Ministère de l'écologie, de l'énergie, du développement durable et de l'aménagement du territoire. Paris.

Tomàs, J.-L., \& Prot, B. (2011). Développement de l'expérience et développement des concepts : de l'activité syndicale à la production d'un référentiel d'activité. Travail et apprentissages, 6, 150-167.

Vygotski, L. (1934/1997). Pensée et langage. Paris: La Dispute.

\section{RÉSUMÉ}

Ce texte propose une approche de la conception de référentiels de diplômes professionnels, depuis l'analyse de l'activité. À partir d'une étude réalisée avec des enseignants de la conduite et de la sécurité routière, il met à l'épreuve l'hypothèse qu'un tel référentiel peut être fondé sur la formalisation de dilemmes spécifiques au genre professionnel. Le texte présente d'abord quelques éléments du contexte institutionnel dans lequel deux études ont été réalisées de 2006 à 2009, avant de préciser la fonction que les chercheurs ont voulu à tenir dans le processus d'élaboration du référentiel, avec les professionnels qui ont été associés. L'analyse de deux séquences d'activité permet ensuite de soutenir l'hypothèse. 


\section{MotS-CLÉS}

Activité, référentiels, dilemmes

\section{RÉFÉRENCEMENT}

Prot, B. (2011). Apprentissage de la conduite et sécurité routière :Un dilemme de référence pour la conception d'un référentiel de diplôme d'enseignant. Activités, 8(2), pp. 189-201, http://www.activites. org/v8n2/v8n2.pdf

Article soumis le 9 mai 2011, accepté pour publication le 8 septembre 2011 